\title{
Ecological effectiveness as an essential quality requirement of innovational construction
}

\author{
Tamara Mkrtchyan ${ }^{1,},{ }^{,}$Ekaterina Lokhova ${ }^{2}$ \\ ${ }^{1}$ Saint Petersburg State University of Industrial Technologies and Design,BolshajaMorskaja str., \\ 23,Saint Petersburg, 191186, Russia \\ ${ }^{2}$ Saint Petersburg School of Economics and Management,Kantemirovskaya str., 3, Saint Petersburg, \\ 194100, Russia
}

\begin{abstract}
Nowadays it seems to be very essential to develop national construction industry by implementation of innovative technologies. As an object of innovational construction «Smart house» or «green building» demonstrates the latest advances in ecological building materials, energysaving structures and lean-technologies. According to the concept of TQM «green building» has tube compatible with environmental protection, environment sustainability and ecological effectiveness requirements. «Green certification» includes these terms into the National environment sustainability rating system. The main goal of this research work was to review and compare theoretical models and concepts of effectiveness and analyze their applicability to determine the term ecological effectiveness as the main Quality Score.
\end{abstract}

\section{Introduction}

The concept of ecological effectiveness is relatively recently included in the concept of economical effectiveness with the development of Green certification system. Such treatment of effectiveness was caused the fact that ensuring of environment sustainability is becoming an important requirement of the market to the company.

Efficiency as a complex concept is being considered by many authors from different points of view such as microeconomic, macroeconomic, operational management, human research management, financial, strategic management. Lance Berger and Dorothy Berger [1] consider the effectiveness as the level of work or action productivity combined with costs. Firstly, efficiency is implicitly connected with the productivity of work or action. Secondly, it is associated with economy, i.e. minimum costs to perform this work or action. It is impossible to characterize efficiency merely by productivity because the result may be obtained, but it would not be the best possible result. Economy also does not characterize efficiency because minimum costs are possible with poor results. Hansen and Wernerfelt [2] propose the model of company efficiency based on several sets of performance variables such as industry indicators, factors relating to its competitors and firm variables.

Reducing production costs through the mastering the techniques of effective production management is the only way to improve the management strategy. So the main aim is to

*Corresponding author: Mkrtchyan75@mail.ru 
identify the factors which have an impact on enterprise efficiency and to find basic manufacturing optimization systems.

The definition of the term "efficiency" includes a number of components. Therefore, a large number and growing body of literature have investigated efficiency increase factors as well as methods and tools for improving performance. The improvement of production efficiency is a complex economic problem of great importance for the economy of an enterprise. This statement is based on the fact that efficiency of production and economic activity leads to the production increase, lower production costs and, thereby, creates the prerequisites for ensuring its competitiveness. The growth of products' competitiveness, in its turn, helps to increase the sales volume and, on this basis, the growth of business profits. Looking ahead, it is obvious that the implementation of manufacturing systems is necessary for the improvement of enterprise efficiency. This part of the work presents an analysis of main tools and methods for enterprise operation optimization.

The Belekoukias et al [3] analyze the relationship between lean methods and tools such as Just-in-Time, Autonomation, Kaizen, Total Productive Maintenance (TPM) and Value Stream Mapping (VSM) and enterprise operational performance. The research reports the study of 140 companies which implement all the lean methods concerned. The authors review works of academicians who had previously explored the lean manufacturing and its impact on the performance and find their studies to be inconclusive. Belekoukias et al [3] research the impact of lean methods application on a number of explanatory variables such as quality, speed, dependability, flexibility and cost. Totality of these variables presents the overall operational performance. The authors conclude that most of the lean methods are correlated with the enterprise efficiency.

The article of Fullerton et al [4] presents the similar point of view on lean manufacturing and firm performance. This paper reports a study of 244 companies. In particular, the research is connected with small, medium and large-sized U.S. manufacturing. The key terms of the article are management accounting practices (MAP) and lean. Management accounting practices are associated with the methods of workforce management and lean MAP provide financial control, essential for informal decision making in lean enterprise. Concerning the term 'lean', the authors represent the lean thinking as the most important strategy for achieving world-class performance. They particularly include practices such as Just-in-Time, Total Quality Management and Total Production Management.

The authors review empirical research in literature which examines the effectiveness of lean management accounting practices strategy. These works admit the authors to come to some hypotheses for their research. Fullerton [4] et al set out to investigate 8 hypotheses which are directly connected with positive relation of lean manufacturing strategy implementation, management accounting practices, value stream costing to visual, operational and financial types of performance.

Another important article is connected with three main areas of the topic of this paper. Bamber [5] et al continue the topic of lean practices, but their study is based on the theory of human research management. They identify the relations between lean management, human resource management and employee efficiency.

Bamber et al [5] analyze the established literature on how different industries cut costs, improve quality and increase overall efficiency in recent decades. The review shows the main process improvement techniques; there are lean tools among them, namely Toyota Production System and Six Sigma. Many of given definitions and various terms are precisely explained, what makes reading clear and understandable. Two systems among the mentioned may be defined as the key terms of this article. Toyota Production System was developed to improve production efficiency through identifying and reducing non-valueadding manufacturing stages. The effect of Six Sigma is similar to Toyota System: it is the 
use of the quality control to cut the number of manufacturing defects by dint of 6 established standards.

The main components for improving efficiency are viewed in details in the article of DubeyaandGunasekaran [6] who examine these factors as constituents of total quality management system. In other words, the authors of this article carry out a study of relations between quality management (in particular, human resources, culture of quality increasing, *motivational leadership and relationship with external and internal partners) and efficiency.

The research findings suggest that quality management dimensions are positively referred to the organizational performance. These results confirm many basic theoretical literature works, but they do not contain enough exploration of the impact of various organizational cultural factors on performance and efficiency. Thereby, Dubeya and Gunasekaran[6]state that there has been a lack of empirical research concerning quality management and performance. Since this problem has not been thoroughly analyzed, the survey of these authors requires a reasonable explanation of the impact of total quality orientation on the performance.

The research methods of Dubeya and Gunasekaran[6] are confirmatory factor analysis and multiple linear regression analysis. The authors carry out a study of a sample of 220 Indian companies covering various sectors to obtain the necessary for the research data. In particular, the research is connected with small-sized and medium-sized enterprises in manufacturing industries. These research methods are very beneficial and may be considered as a break-through, they throw new relevant light on the subject of the relationships between components of total quality management and performance or efficiency. Comparing to the previous literature works, which contained only empirical data, the choice of quantitative research methods seems to be the most valid, effective and well-grounded.

The chosen research methods allow the authors to identify the correlation between human resources, quality culture, motivational leadership and relationship with external and internal partners and maintenance performance. Altogether the findings indicate significant relations between quality management and organizational performance.

However, there are a lot of empirical works which represent similar results. For instance, Maletic et al [7] reveal positive relations between components of total quality management such as customer orientation, quality responsibility, prevention and performance maintenance in a sample of 53 Slovenian companies in manufacturing, construction and transportation industries. The paper of Challis et al (2005) reports on the relationships between various organizational sectors (such as leadership, teams, human resource management and benchmarking) and enterprise efficiency. The results of this study show that all of these components are associated with company performance, but human resources have significant relation to overall efficiency.

Another point worthy of consideration is the article of Haines et al [8]. The authors carry out a study of 312 private and public sector companies. They aim to use a questionnaire to draw attention upon the key elements and relationship between human research practices and performance management efficiency. They examine three main variables of human research management such as culture, climate and strategic integration of human research management and suppose that these elements are positively related to production efficiency.

To support the main idea of this work Haines et al [8] choose the research method of hierarchical multiple regressions. For collecting data, they developed a questionnaire which was mailed to managers of human resource departments in companies with 200 and more employees in Canada. The questionnaire includes 8 main parts which are related to performance management practices, such as trainings and multisource feedback, 
organizational culture and employee relations climate. The developing of this form is a good start for the findings and it gives a solid amount of significant empirical data for regression models.

On the one hand, this article is coherent. Furthermore, it presents a wide range of references which means that authors' statements are not merely a product of their imagination, but they are supported in the literature. On the other hand, the findings of this study should not be considered to be weighty for their field because this paper provides little evidence of complex effects of practices and efficiency. During the literature analyzing it was noticed that one of the most successful methods to increase the efficiency of a producing company is to introduce lean manufacturing (Amin and Karim, [9]). As a consequence, among the methods supposed to be applied for product line improving there are conceptions of lean manufacturing such as ' 5 -S', Just-in-Time, Total Productive Maintenance, Kaizen and other systems. However, it is difficult to distinguish the systems which can help to scheme an effective production strategy at this stage; the necessary results will be obtained only after the stage-by-stage analysis of company's activity and evaluation of each of the lean manufacturing methods listed.

As many authors claim, the work of employees has a great influence on the general efficiency of a company[9]. Therefore, the study considers not only correct labour division, qualification of employees and their working regime, but also the motivation aspects of their working activity. It is supposed to conduct a survey among the primary production workers aiming to determine the key factors which influence productivity. In this case exploratory factors will be applied for the analysis with the purpose of identifying any possible relations between measured variables. According to this method, factors which have the weakest efficiency effect may be excluded. Confirmatory factor analysis allows identifying relationship between different factors and enterprise efficiency. Correlation and regression analyses help to determine the direction and the measure the relations. Furthermore, this part involves calculation of the following characteristics: an average monthly number of workers, labor resources fund, standard number of workers for the performance of shift's production target, etc[10].

One concludes that there is a complex system of efficiency increase factors. In general, this system includes the main components of total quality management such as the quality culture, the relationship with external and internal partners, the role of managers and leadership and the last significant factor - human resources.

\section{Methodology}

Over the past decades ensuring stable operation of an enterprise producing competitive products is the basic purpose for managers at all levels. The history has a lot of examples of large foreign companies which achieved success by lower production costs, rational usage of material resources, achieving higher economic performance and increasing productivity and efficiency. Until recently, the increasing economic efficiency was the most important and urgent task of production management. But nowadays, the companies' proposal has to be compatible with environmental protection, environment sustainability and ecological effectiveness requirements. The above-mentioned definitions as the components of ecological effectiveness and appropriate managerial processes, which aimed at ensuring the high standard of living, cover different spheres of human being. Therefore, specified analysis of the principal elements of ecological effectiveness requirements' global approach deserves to be observed. We are especially concerned about the application approach considered towards national construction industry which seems to be very essential to be developed by. 
In the Russian quality standards system ecological effectiveness is recently used as a criterion of the object in question. This way of development has been implemented by specialists on certification of the construction industry, which is committed to international standards and implement ambitious projects such as "green building" in the global concept of "Smart City." This direction of development of the construction industry is becoming the paradigm of innovation development of the sector, it is the embodiment of construction industry tomorrow.

Projects of "green building" are maximally aimed at preserving the eco-system. They ensure consumers harmoniously exist throughout life to the environment. Customer value this kind of construction products is largely mediated by using resource-saving ecotechnology, innovative methods to achieve energy efficiency, environmental friendliness and comfort, taking into account a lean approach to natural resource consumption.

"Green building" - a new product with a new customer value, which is positioned as a human environment. This statement was included in the Russian standards with the filing of recognizing world systems such as LEED, BREEAM. System of standards in construction this type of complexes is in its infancy and is constantly updated with new terms, concepts, and regulatory elements. National "Green certification" system is developed and used, for example: GOST R 54964-2012 "Conformity assessment. Environmental requirements to the objects of real estate, "STO NOSTROY 2.35.68-2012" Green Building ". Residential and public buildings. Ratings system of assessment environment sustainability system. " Green building object is interpreted in the standard as a habitat, ensuring the effectiveness of green building object is understood as building capacity to ensure environment sustainability. In the standard is developed the corresponding rating system of ecoefficiency and appropriate indicators of economic efficiency. Objects of green building are much more expensive than the objects are made in accordance with the usual standards of construction that is not evidence in favor of "green."Only the increment of green building value in exchange for guarantees of eco-efficient best practices application in its design and construction has not deterred consumers. It means that the criterion of eco-efficiency is becoming a significant component of the objects competitiveness [11], its understood as the ability of the economic entity to maintain the environment sustainability of the company.

The inclusion the environmental effectiveness criteria of the customer value concept, prescribed by the Russian national "green standards", is a proof of a certain consistency and maturity of an integrated quality management system in the Russian Federation. The quality of the environment, which forms the quality of life is spelled out in the standards, is developed and constantly supplemented operating procedures certification system which is accrediting manufacturers to ensure that they comply with and maintain the required level.

Thus, the process of TQM concept evolution and an integrated approach to the quality predestined transition from the concept of "efficiency" to the concept of eco-efficiency. Today, eco-efficiency is a part of the National environment sustainability rating system[13,14]. It becomes an important element of the rating attractiveness of the project that contributes the level of business reputation, prestige and social responsibility of its participants.

Certification Systems evaluating vendors on environmental performance criteria are causing the consumer a high level of confidence, as the reliability of these systems mediate manufacturer and guarantee the environment sustainability. The National environment sustainability rating system treats the stability of the environment as a set of quality characteristics of the building and surrounding areas, characterize the comfort of human life in harmony with the environment. In this meaning of "green standard" a modern building should be well blend into the surrounding ecosystem, becoming an integral part thereof. The concept of «smart house» in the modern sense is connected to design, to build and to operate the construction with 
taking into account a lean approach. Thus, the technology of lean production and consumption are updated for the construction industry through the systematic introduction of certification systems. "Green standards" have positioned the building as a human environment, thus laying the essence of the "smart home" - a combination of the comfort criteria, energy efficiency, environmental friendliness and innovative resource-saving technologies. "Smart House" - a thrifty house. It is already being laid at the design stage rather expensive technologies, which leads to higher project costs, but the subsequent operation of the building will be environmentally friendly and economical. It employs the basic principle of lean approach - overpay initially to have quality resources, and then save on the final yield level, commissioning, start-up complex of all associated equipment and subsequent maintenance.

\section{Conclusion}

We analyzed the applicability 'effectiveness' methodological concepts to determine 'ecological effectiveness' as the essential demand on the quality of 'green construction'. Our investigation was targeted by Green certification' requirements for national construction industry. We observed the components of ecological effectiveness' term and appropriate managerial processes which aimed at ensuring the high standard of living at the international rate. Hereby we attempted to observe 'The National environment sustainability rating system' in order to determine principal 'ecological effectiveness 'quality scores. We found that the eco-efficiency in this sense is identical with economic efficiency, expressed in classical technical-economic indicators. Understanding this relationship becomes apparent as the producers in the sector - construction companies, end users "smart home" and to potential investors, who are beginning to admire environment quality and life quality and pay them more attention. Cultivating consumer interest in "smart homes" with their inherent potential contributes to the development of certification systems are operating on the basis of "green standards". These standards offer serious prospects for the construction industry development.

\section{References}

1. L.A. Berger, D.R. Berger, The Compensation Handbook: A State-of-the-art Guide to Compensation Strategy and Design(McGraw-Hill, New York,2010)

2. G.S. Hansen, B. Wernerfelt, Strategic Management Journal 10(5), 399-411(2010)

3. I.Belekoukias, J.Garza-Reyes, V.Kumar, International Journal Of Production Research 52(18), 5346-5366 (2014)

4. R.R. Fullerton, F.A. Kennedy, S.K. Widener, Journal Of Operations Management 32(7/8), 414-428(2014)

5. G.Bamber, P.Stanton, T.Bartram, R.Ballardie, International Journal Of Human Resource Management 25(21), 2881-2891 (2014)

6. R.Dubey, A.Gunasekaran, International Journal Of Production Research 53(2), 371382(2015)

7. D.Maletic, M.Maletic, B.Gomiscek, International Journal Of Production Research 52(6), 1744-1754(2014)

8. I.V. Haines, S.St-Onge, International Journal Of Human Resource Management 23(6), 1158-1175(2012) 
9. M.A. Amin,M.Karim, International Journal Of Production Research51(4), 11461167(2013)

10. D.Challis, D.Samson, B.Lawson, International Journal Of Production Research 43(1), 81-107(2005)

11. Russian Standard STO NOSTROY 2.35.68-2012

12. Experts respond AVOK №1'2012

13. S. Kuzmina, Saint-Petersburg Economic Journal 4, 145-154 (2016)

14. V.V.Okrepilov, S.N.Kuzmina, V.L.Makarov, A.R.Bakhtizin, Economy of Region 2, 301-313 (2015) 